\title{
Adjuvant Chemotherapy in Resected Biliary Tract Cancer
}

\author{
John N. Primrose ${ }^{1,2}$ \\ ${ }^{1}$ University of Southampton, Southampton, UK; ${ }^{2}$ University Surgery, Mailpoint 816, C Level South Academic Block, \\ University Hospital Southampton, Southampton, UK
}

For many years, biliary tract cancer was something of a 'Cinderella' malignancy. It appeared highly lethal, presented late, the surgery was difficult with high levels of morbidity, and there was little pharma interest as it was a less common cancer. Before the last decade, there were no definitive reports on the use of chemotherapy that could be used to guide the management of patients with any confidence. The only adjuvant trial of note covered pancreatic cancer as well as all biliary cancer, ${ }^{1}$ and, although it showed some potential in gallbladder cancer, the chemotherapy schedule was not one that is presently used.

Between 2005 and 2015, a number of groups began to take a serious interest in biliary tract cancer. The UK National Cancer Research Institute (NCRI) initiated an advanced disease study (ABC01/2) with gemcitabine/cisplatin as the experimental arm and gemcitabine alone as the control. ${ }^{2}$ An adjuvant trial (BILCAP) was also launched using capecitabine as the experimental arm and a notreatment control arm. ${ }^{3}$ The basis of this choice, fortunate in retrospect, was that there was no standard treatment on which the adjuvant regimen could be based. It was thought that in the event of relapse, the patient could be recruited to $\mathrm{ABC} 02$. The $\mathrm{ABC} 01 / 2$ trials recruited quickly and, in $2010,{ }^{2}$ reported a significant survival benefit for the combination treatment, establishing a standard of care in advanced biliary tract cancer. Interestingly, despite many other schedules being trialled, this combination remains the

(C) Society of Surgical Oncology 2020

First Received: 21 April 2020;

Published Online: 6 May 2020

J. N. Primrose

e-mail: j.n.primrose@ soton.ac.uk first-line standard of care to this day. The NCRI group has also undertaken a second-line study that has also produced a standard of care in this setting. ${ }^{4}$

In addition to BILCAP, a number of other groups turned their attention to adjuvant therapies. The Nagoya group (Japan) launched the BCAT study in 2007, which compared gemcitabine alone as adjuvant with surveillance. The results of this trial were reported in $2018 .^{5}$ Only extrahepatic cholangiocarcinoma, not intrahepatic or gallbladder cancer, were examined in this trial. The French Group (PRODIGE 12-ACCORD 18-UNICANCER GI) opened a study in 2009 comparing gemcitabine/oxaliplatin with surveillance as adjuvant in all biliary tract cancer; this trial reported in 2019. ${ }^{6}$ In 2013, the Japan Clinical Oncology Group launched JCOG1202 (ASCOT study) ${ }^{7}$ examining the use of S1, an orally available fluoropyrimidine, versus surveillance in all biliary cancer. This trial has completed recruitment but has not yet reported. Lastly, in 2015, an international intergroup study opened (ACTICCA-01), which set out to examine the use of the $\mathrm{ABC} 02$ regimen (gemcitabine/cisplatin) versus observation. ${ }^{8}$ As discussed below, the design of this study has been revised.

The role of adjuvant therapy in biliary tract cancer has been comprehensively reviewed recently. ${ }^{9}$ In summary, it is useful to split the adjuvant schedules into gemcitabinebased and fluoropyrimidine-based regimens. The two reported gemcitabine-based studies have both been negative. The BCAT $^{5}$ study was completely negative, with no difference between the arms. PRODIGE $12^{6}$ was also reported as negative, although there was a trend towards benefit, with a non-significant hazard ratio of 0.88 for progression-free survival. The trial may have been underpowered, with only 196 patients being recruited. Of the fluoropyrimidine-based studies, only BILCAP has reported. ${ }^{3}$ The study demonstrated that the overall survival (OS) improved by approximately 16 months, from 36.4 in the control arm to 51.1 months in the capecitabine arm. This 
escaped significance on the intention-to-treat analysis, but was positive in the per protocol analysis (excluding patients who did not receive the allocated capecitabine). It was also significant on the preplanned sensitivity analysis, and the difference in PFS was significant. On the basis of the large effect size, capecitabine has been recommended as standard of care in the American Society of Clinical Oncology (ASCO) guidelines. ${ }^{10}$ The ASCOT study has completed recruitment but has not yet reported. The results are also keenly awaited, not least for the fact that it will be possible to meta-analyse the results with BILCAP, as capecitabine and S1 are similar drugs. For the immediate future, ACTICCA-01 has changed design to compare gemcitabine/cisplatin (the standard of care in advanced disease) with capecitabine, which is now the standard of care adjuvant drug. The trial is continuing after its interim analysis, therefore there is no suggestion of harm in the experimental arm.

In this issue of Annals of Surgical Oncology, Seita et al. ${ }^{11}$ have investigated the adjuvant treatment of biliary tract cancer, but have taken a different approach. The authors are from the same Nagoya group that performed the highly successful and informative, although negative, BCAT trial. This is a phase IIa (non-randomized) study recruiting what they consider to be a higher risk group, i.e. those with node-positive disease. This is an interesting although high-risk strategy. While it is possible that the effect size might be greater, recruitment would be much lower than if all patients were included. However, the group feel they can identify patients in whom the risk of relapse is low and hence they may not benefit from adjuvant treatment. In this study, the researchers have chosen to use S1 as therapy, which, as stated, is the agent used in the JCOG1202 randomized trial that has completed recruitment.

The study itself planned to recruit 50 patients. As it was, non-randomized historical controls were used to assess the effect size. Data suggested that the 3-year OS would be around $30 \%$ in this cohort. It was anticipated that in the study, a 45\% 3-year OS might be achieved. In fact, a 50\% 3-year OS was achieved, and, by the criteria of the investigators, this represents, in their view, a 'signal' that S1 may be effective in this setting. It is possible to make a comparison with the data from the only other adjuvant study using a fluoropyrimidine, namely BILCAP. ${ }^{3}$ Although the 3-year OS was not an endpoint in BILCAP, it can be derived. The subgroup analysis paper from BILCAP is unpublished but is in preparation. It is certainly the case that OS in the node-negative patients is very high and this accords with the authors contention. Similarly, the OS in the node-positive patients is much lower, as reported in the BILCAP manuscript. The benefit of chemotherapy appears in fact to be in the same order as reported by Seita et al. ${ }^{11}$, and gives support to the findings. Looking at the tolerability and toxicity of S1, there is no real surprise, and, again, is broadly similar to BILCAP. A tentative conclusion might be that a 'signal' of efficacy is evident in this study.

There are however several caveats. The main weakness of the approach of this study is that it is not randomized. Where possible, phase II trials should be randomized. The aim of a phase II trial is to determine if there is enough evidence of efficacy to investigate in a phase III study. In the case of an advanced cancer, it is possible a signal can be obtained by seeing a response in a non-randomized study if the effect size is large enough, but, even then, randomization is better. However, in adjuvant studies, the endpoint is relapse, therefore the only comparator is retrospective data. This can be highly unreliable. Patients who endure a major operation and then are quickly fit for chemotherapy are a highly selected group who will have a better outlook than the average patient. This was certainly the case in BILCAP, where the power calculation had to be revised as both arms of the trial were surviving better than historical controls.

In conclusion, it may be that $\mathrm{S} 1$ has potential as an adjuvant in resected biliary tract cancer, but the data from this trial are insufficient to confirm this with certainty. It gives support to the purpose of JCOG1202 (ASCOT study $)^{7}$ and the result of this trial is awaited with great interest. However, to give an unequivocally robust answer as regards the role of fluoropyrimidines in biliary tract cancer meta-analysis will almost certainly be required of all appropriate studies. It is unfortunate that this present trial cannot be included in any such analysis as it lacks an observation-only control arm.

\section{REFERENCES}

1. Takada T, Amano H, Yasuda H, Nimura Y, Matsushiro T, Kato $\mathrm{H}$, et al. Is postoperative adjuvant chemotherapy useful for gallbladder carcinoma? A phase III multicenter prospective randomized controlled trial in patients with resected pancreaticobiliary carcinoma. Cancer. 2002;95:1685-1695.

2. Valle J, Wasan H, Palmer DH, Cunningham D, Anthoney A, Maraveyas A, et al. Cisplatin plus gemcitabine versus gemcitabine for biliary tract cancer. $N$ Engl $J$ Med. 2010;362:1273-1281.

3. Primrose JN, Fox R, Stubbs, C, Neoptolemos J, Garden OJ, Valle $\mathrm{J}$, et al., for the BILCAP Study Group. Adjuvant capecitabine for resected biliary tract cancer. The BILCAP randomised trial. Lancet Oncol. 2019;35:663-673.

4. Lamarca A, Palmer DH, Wasan HS, Ross PJ, Ting Ma Y, Arora A, et al: ABC-06|A randomised phase III, multi-centre, openlabel study of Active Symptom Control (ASC) alone or ASC with oxaliplatin/5-FU chemotherapy (ASC + mFOLFOX) for patients (pts) with locally advanced/metastatic biliary tract cancers (ABC) previously-treated with cisplatin/gemcitabine (CisGem) chemotherapy [abstract no. 4003]. J Clin Oncol. 2019;37(15 Suppl). 
5. Ebata T, Hirano S, Konishi M, Uesaka K, Tsuchiya Y, Ohtsuka $\mathrm{M}$, et al. Randomized clinical trial of adjuvant gemcitabine chemotherapy versus observation in resected bile duct cancer. $\mathrm{Br}$ J Surg. 2018;105:192-202.

6. Edeline J, Benabdelghani M, Bertaut A, Watelet J, Hammel P, Joly JP, et al. Gemcitabine and oxaliplatin chemotherapy or surveillance in resected biliary tract cancer (PRODIGE 12-ACCORD 18-UNICANCER GI): a randomized phase III study. J Clin Oncol. 2019;37:658-667.

7. Nakachi K, Konishi M, Ikeda M, Mizusawa J, Eba J, Okusaka T, et al., on behalf of the Hepatobiliary and Pancreatic Oncology Group of the Japan Clinical Oncology Group. A randomized phase III trial of adjuvant S-1 therapy vs observation alone in resected biliary tract cancer: Japan Clinical Oncology Group Study (JCOG1202, ASCOT). Jpn J Clin Oncol. 2018;48(4):392-395.

8. Stein A, Arnold D, Bridgewater J, Goldstein D, Jensen LH, Klumpen HJ, et al. Adjuvant chemotherapy with gemcitabine and cisplatin compared to observation after curative intent resection of cholangiocarcinoma and muscle invasive gallbladder carcinoma (ACTICCA-1 trial): a randomized, multidisciplinary, multinational phase III trial. BMC Cancer. 2015;15:564.

9. Lamarca A, Edeline J, McNamara MG, Hubner RA, Nagino M, Bridgewater J, et al. Current standards and future perspectives in adjuvant treatment for biliary tract cancers. Cancer Treat Rev. 2020;84:101936.

10. Shroff RT, Kennedy EB, Bachini M, Bekaii-Saab T, Crane C, Edeline $\mathrm{J}$, et al. Adjuvant therapy for resected biliary tract cancer: ASCO clinical practice guideline. $J$ Clin Oncol. 2019;37(12):1015-1027.

11. Seita K, Ebata T, Mizuno T, et al. Phase II trial of adjuvant chemotherapy with S-1 for node-positive biliary tract cancer $(\mathrm{N}$ SOG 09). Ann Surg Oncol. 2020. https://doi.org/10.1245/s10434020-08364-2.

Publisher's Note Springer Nature remains neutral with regard to jurisdictional claims in published maps and institutional affiliations. 